\title{
A DESIGN METHOD OF LOGICAL TOPOLOGY FOR IP OVER WDM NETWORKS WITH STABLE ROUTING
}

\author{
Junichi Katou \\ Department of Informatics and Mathematical Science \\ Graduate School of Engineering Science, Osaka University \\ 1-3 Machikaneyama, Toyonaka, Osaka 560-8531, Japan \\ j-katou@ics.es.osaka-u.ac.jp
}

\author{
Shin'ichi Arakawa \\ Graduate School of Economics, Osaka University \\ 1-7, Machikaneyama, Toyonaka, Osaka 560-0043, Japan \\ arakawa@ics.es.osaka-u.ac.jp
}

\author{
Masayuki Murata \\ Cybermedia Center, Osaka University \\ 1-30, Machikaneyama, Toyonaka, Osaka 560-0043, Japan \\ murata@cmc.osaka-u.ac.jp
}

\begin{abstract}
An IP (Internet Protocol) over WDM network is expected to be an infrastructure for the next-generation Internet by directly carrying IP packets on the WDM-based network. Among several architectures for IP over WDM networks, one promising way is to overlay a logical topology consisting of lightpaths over the physical WDM network, so that IP packets are carried using the lightpaths. The conventional design methods of the logical topology have been focusing on maximizing throughput of the traffic. However, when the WDM network is applied to IP, the end-to-end paths provided by the logical topology of the WDM network is not suitable to IP routing since IP has its own metrics for route selection. We therefore propose a new heuristic algorithm to design a logical topology by considering the delay between nodes as an objective metric. We use a non-bifurcated flow deviation method to obtain a set of routes that IP packets are expected to traverse. Our proposal is then compared with conventional methods in terms of the average packet
\end{abstract}


delays and throughput. It is shown that our method becomes effective when the number of wavelengths is a limited resource.

Keywords: IP over WDM, logical topology, flow deviation, route stability

\section{INTRODUCTION}

IP (Internet Protocol) over WDM networks where IP packets are directly carried over the WDM network is expected to offer an infrastructure for the next generation Internet. A currently available product for IP over WDM networks is only for providing the large bandwidth on point-to-point links. That is, each wavelength on the fiber is treated as a physical link between the conventional IP routers. In this way, the link capacity is certainly increased by the number of wavelengths multiplexed on the fiber, but it is insufficient to resolve the network bottleneck against an explosion of traffic demands since it only results in that the bottleneck is shifted to an electronic router.

One promising way to alleviate the bottleneck is to configure the wavelength paths over the WDM physical network and to carry IP packets utilizing the wavelength paths. Here, the physical network means an actual network consisting of the optical nodes and optical-fiber links connecting nodes. Each node has optical switches directly connecting an input wavelength to an output wavelength, by which no electronic processing is necessary at the node. Then, the wavelength path can be set up directly between two nodes via one or more optical switches. Hereafter, we will call the wavelength path directly connecting two nodes as a lightpath.

By utilizing the logical topology consisting of lightpaths, the physical structure of the WDM networks is embedded, and the logical topology is viewed as a underlying network by IP. In such a network, if the lightpaths are placed between every two end nodes, then no electronic processing is necessary within the network. However, too many wavelengths are necessary to establish such a network [Ramaswami and Sivarajan, 1995]. By limiting the number of lightpaths, on the other hand, we need less wavelengths though a routing capability should be provided at nodes, which will be described in more detail in the next section. In this approach, lightpaths are first established by using the available wavelengths as much as possible. If the direct lightpath cannot be set up between two nodes, two or more lightpaths are used for packets to reach the destination.

Many researchers have discussed the design methods of the logical topology. See, e.g., [R.Dutta and G.N.Rouskas, 2000] and references 
therein. For example, the authors in [Mukherjee et al., 1996] formulate a design method of the logical topology as an optimization problem, and show that the problem is NP-hard. In [Ramaswami and Sivarajan, 1996], the authors combine the logical topology design problem and routing problem so as to maximize the network throughput. Since the combined problem is computationally hard to solve, they split it into two subproblems, and solve those two subproblems independently. The routing problem is formulated as a linear programming problem by imposing the delay constraint for each node pair. Several heuristics are also proposed to relax the computational burden.

We should note here that MPLS (Multi-Protocol Label Switching) is now being developed by IETF [R.Callon et al., 2000, Jamoussi, 2000, R.Callon et al., 1999], and is considered to be applied to IP over WDM networks [D. O. Awduche and Coltun, 2000]. Among several options of MPLS, the route the packet traverse may be determined explicitly (explicit routing). In such a network, the lightpath should be prepared among every end node pairs within the MPLS domain, which requires too many wavelengths as described in the above. To alleviate the problem, we split the lightpath within the network. In this approach, it may take two or more lightpaths within the IP over WDM network for the packets to be forwarded. Then, the IP routing capability becomes necessary within the network. See Section 2 for more detail.

In our network, packet route is determined by the routing protocol provided by the IP layer, and the WDM network only provides (logical) paths between nodes. Then, in designing the logical topology, routes of the lightpaths should be determined by considering the nature of the IP routing protocol. That is, we place lightpaths such that the IP packet experiences smaller delays on its end-to-end path as much as possible. For this purpose, we try to reduce the number of (electronic) nodes in addition to small propagation delays between two end nodes.

A routing stability of IP is another important issue in designing IP over WDM networks. Most of conventional researches assume the amount of traffic between nodes are given and fixed. In building IP networks, however, the issue on routing stability should also be considered. In our experiments, we compare the delays of first and second shortest endto-end paths, and if packet delays experienced by those two paths are different, we will conclude that the logical topology is "robust" against the traffic fluctuation. Actually, we will show through numerical examples that our proposed method is robust against the routing stability.

The paper is organized as follows. In Section 2, we describe our architecture model of optical node. In Section 3, we propose the logical topology design method considering the route stability. A flow deviation 
method, one of methods for flow assignment on the logical topology, is shown in Section 4. In Section 5, we compare and evaluate our proposed algorithm with the conventional algorithm. Finally, Section 6 concludes this paper.

\section{ARCHITECTURAL MODEL OF NODES}

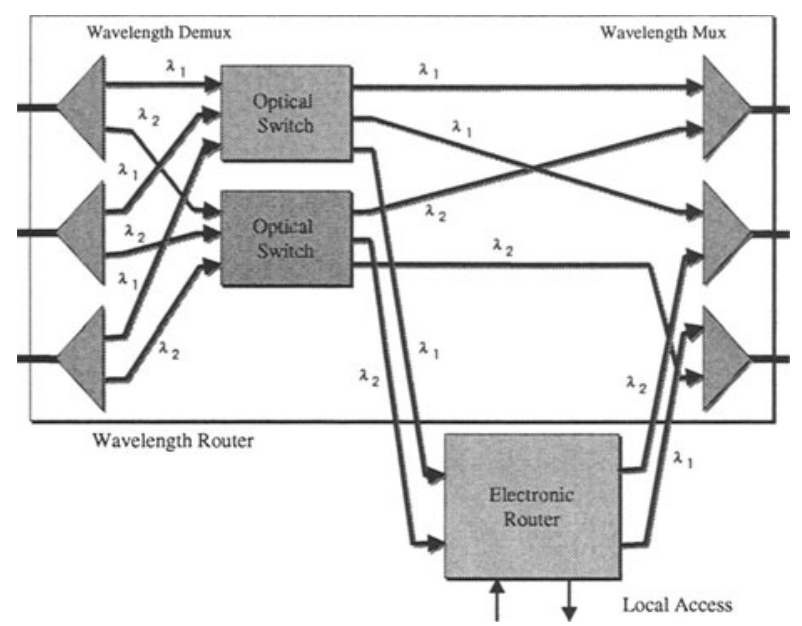

(a) Node Architecture

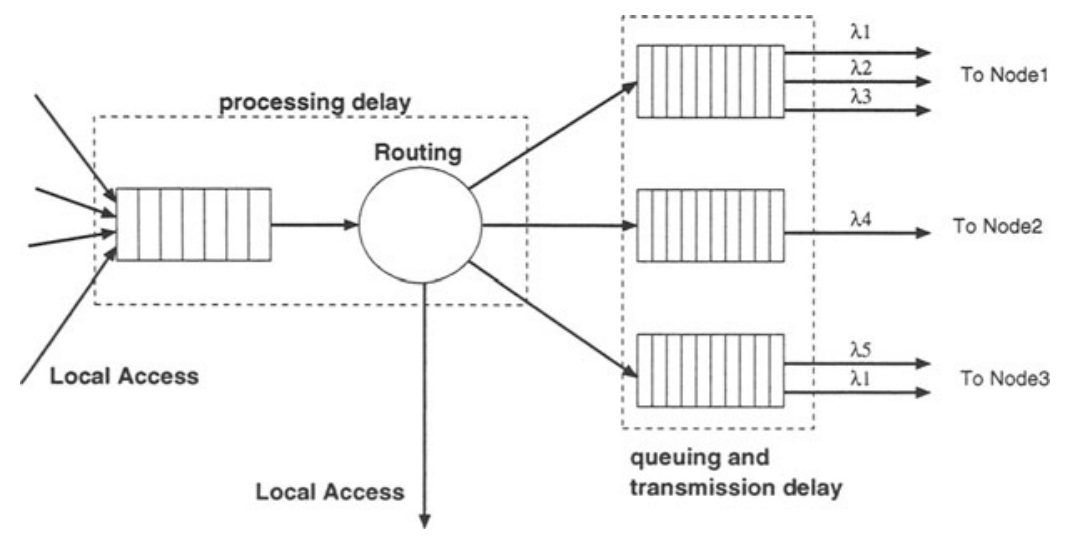

(b) Model of Electronic Router

Figure 1 Node Architecture Model 
Figure 1 shows our architecture model of the optical node. Every node is equipped with an optical switch and an electronic router. The optical switch consists of three main blocks; input section, a non-blocking switch, and output section. At input section, optical signals are demultiplexed into $W$ fixed wavelengths, $\lambda_{1}, \ldots, \lambda_{w}$. Each wavelength is switched into an appropriate output port at non-blocking switch without wavelength changes. Finally, wavelengths on the fiber are again multiplexed, and go to the next node. Note that a lightpath is placed by configuring the non-blocking switch along the path, so that the traffic on a particular wavelength from the input port to the output port is forwarded without any electronic processing.

As having been described in the previous section, all end node pairs are not always provided a one-hop lightpath in our target system, by which the use of wavelengths can be reduced. If the lightpath is terminated at the node, then IP packets on that lightpath is converted to electronic signals and forwarded to the electronic router. The electronic router processes the packet forwarding, just same as the conventional routers. If the packet should be further forwarded to other nodes, it is put on the adequate lightpath.

In our study, an electronic router is modeled as shown in figure 1(b). IP packets, which come from an optical switch or local access, are first buffered for processing, and then these packets are processed on FIFO (First In First Out) basis. In the case where the packets are forwarded onto the network, those are queued on the appropriate output port buffer. In this paper, we assume that multiple lightpaths between an adjacent node pair share the same buffer.

We last note that the other structures of optical nodes can also be considered, but the above-mentioned node architecture is preferable since there is no need to modify the IP routing mechanism.

\section{LOGICAL TOPOLOGY DESIGN ALGORITHM}

In [R.Ramaswami and Sivarajan, 1995], the authors propose a heuristic algorithm called MLDA (Minimum delay Logical topology Design Algorithm) to establish a logical topology. MLDA works as follows. First, it places the lightpath between two nodes if there exists a fiber directly connecting those nodes. Then, attempts are made to place lightpaths between nodes in the order of descending traffic demand. Finally, if there still exist non-utilized wavelengths, lightpaths are placed randomly utilizing those wavelengths as much as possible. We again note that a lot of conventional methods including MLDA are focusing mainly 
on maximizing throughput of traffic and those are not adequate to design a logical topology suitable to carry IP traffic.

We therefore introduce a new logical topology design algorithm called SHLDA (Shortest-Hop Logical topology Design Algorithm) to resolve the above-mentioned problems. As described before, we assume that a routing function is performed only at the IP layer. Then, the logical topology should be designed by incorporating the nature of route selection adopted in the IP routing protocol. It is natural that a shorter path would be selected by the IP routing protocol for forwarding packets. (We note here that by a short path, we mean that the number of lightpaths between an end node pair is small.) That is, hop counts of lightpapths (i.e., the number of lightpaths that the packet traverses) should be reduced as much as possible, which is our primary objective in the proposed algorithm. Once the lightpath is split between some two end node pair, and a series of lightpaths is necessary to reach the destination, the processing delay at the electronic router must be considered. It will be incorporated in the final determination of the lightpaths setup, which will be described in the next subsection.

MLDA uses traffic demand between node pairs to set up the next lightpath in the algorithm. On the contrary, we use the performance metric $F_{i j}$ for node pair $i j$, which is determined by the following equation,

$$
F_{i j}=\gamma_{i j} \times h_{i j}
$$

where $\gamma_{i j}$ is the traffic demand from node $i$ to $j$, and $h_{i j}$ is the hop-count of the minimum hop route between node $i$ and node $j$ on the physical topology. Here, the hop-count of the lightpath refers to the number of physical links that the lightpath traverses. Note that $F_{i j}$ is equal to $\gamma_{i j}$ in MLDA, i.e., MLDA does not consider the hop-count of the lightpath, and only uses the propagation delay in determining the shortest route of the lightpath.

On the other hand, SHLDA first uses the hop-count as the metric to calculate the shortest route in configuring the lightpath. It enables us to establish the lightpath consisting of the minimum number of electronic routers that IP packets traverse. The detailed description of the SHLDA algorithm is as follows.

Step 1: Calculate the metric $F_{i j}$ for each node pair $i j$ from the traffic matrix $Q=q_{i j}$. In initially determining $F_{i j}$, $h_{i j}$ is simply set as the hop-count of the shortest physical path.

Step 2: Place the lightpath between two nodes if there exists a fiber.

Step 3: Select the node pair $i^{\prime} j^{\prime}$, where $i^{\prime}$ and $j^{\prime}$ are indices giving $\max _{i j}\left(F_{i j}\right)$. If $F_{i^{\prime} j^{\prime}}=0$, go to Step 5 . Otherwise, go to Step 4 . 
Step 4: Find the minimum-hop route between the node pair $i^{\prime} j^{\prime}$, and check the availability of wavelengths in order to configure the lightpath. If so, use the wavelength with lowest index to establish the lightpath. Then set $F_{i^{\prime} j^{\prime}}=0$ and go back to Step 3. If there is no available wavelength, set $F_{i^{\prime} j^{\prime}}=0$ and go back to Step 3.

Step 5: If there still exist non-utilized wavelengths, lightpaths are configured randomly as much as possible utilizing those wavelengths as in MLDA.

By the above algorithm, we obtain the logical topology, but it is insufficient in building IP over WDM networks. We consider the routing stability by next applying the flow deviation method.

\section{FLOW DEVIATION METHOD}

In this section, we first introduce the flow deviation method [L.Fratta et al., 1973] in Subsection 4.1. Then, it is applied to our case in Subsection 4.2 .

\subsection{DESCRIPTION OF FLOW DEVIATION METHOD}

In this subsection, we summarize the flow deviation method [L.Fratta et al., 1973]. It incrementally changes the flow assignment along the feasible and descent direction. It works as follows. Given an objective function $T$, the flow deviation method sets $l_{i j}$ as the partial derivative with respect to $\lambda_{i j}$, where $\lambda_{i j}$ is the flow rate of lightpath(s) between nodes $i$ and $j$. Then, the new flow assignment is solved by using the shortest path algorithm in terms of the $l_{i j}$. By incrementally changing from the old flow assignment to the new one, the optimal flow assignment is explored. The detailed description of the flow deviation method is as follows.

Step 1: Prepare a starting feasible flow assignment $f^{0}$. Let $n=0$.

Step 2: Set $g \leftarrow f^{n}$. Assume that flow assignment $f^{n}$ is represented as $\left\{x_{11}, \ldots, x_{p q}, \ldots, x_{N N}\right\}$.

Step 3: Calculate $l_{i j}=\frac{\partial T}{\partial \lambda_{i j}}$. Then, set the new flow assignment $R(g)$ to $\left\{x_{11}^{\prime}, \ldots, x_{p q}^{\prime}, \ldots, x_{N N}^{\prime}\right\}$ by solving the shortest path algorithm using the metric $l_{i j}$.

Step 4: For each node pair $i j$, do following.

Step 4.1: Let $v$ be the flow assignment by deviating flow $i j$ from $g$ toward $R(g)$. That is, the resulting flow assignment, $v$, is set to $\left\{x_{11}, \ldots, x_{i j}^{\prime}, \ldots, x_{N N}\right\}$. 
Step 4.2: Check whether $v$ is feasible. Feasible $v$ means that the processing capability of IP routers and/or the capacity of a lightpath does not exceed its limit. If not, then the deviation at Step 4.1 is rejected, and go back to Step 4 .

Step 4.3: Check whether the $v$ is descent. If $T(v)<T(g), g$ is allowed to deviating toward $v$. Then, $g \leftarrow v$. Then go back to Step 4. If $T(g) \leq T(v)$, the deviation from the $g$ toward $R(g)$ is rejected, and go back to Step 4 .

Step 5: If $g=f^{n}$, then stop iteration. Note that $g=f^{n}$ means there is no improvement of performance by deviating the flow. Otherwise, set $n \leftarrow n+1$, and go back to Step 2 .

\subsection{DERIVATION OF THE METRIC $l_{\mathrm{v}}$}

In this subsection, we will show the metric $l_{i j}$ in our case. We will use the following notations.

$N$ : the number of nodes in the network

$P_{i j}$ : the propagation delay of lightpath $i j$

$C$ : the capacity of each wavelength

$\mu$ : processing capability at an electronic router, which is assumed to be identical among all routers.

We also introduce the following variables.

$a_{i j}^{s d}$ : when the packets are routed from node $s$ to node $d$ via the lightpath $i j$, the value is set to be 1 . Otherwise, 0 .

$\delta_{i}$ : the sum of all traffic switched by the IP electronic router at node $i$ except the traffic flow $\lambda_{i j}$.

The objective function $T$ in our case is given as the average of $T_{s d}$ 's (the delay between node $s$ and $d$ ), i.e.,

$$
T=\frac{1}{N(N-1)} \sum_{s=1}^{N} \sum_{d=1}^{N} T_{s d}
$$

As shown in figure 1, the delay experienced at a node consists of processing delay and transmission delay. Henceforth, the delay between nodes $s$ and $d$ consists of the propagation delay, processing delay and transmission delay. That is, we have

$$
T_{s d}=\left[\sum_{i j} a_{i j}^{s d} P_{i j}\right]+\left[\sum_{i j} a_{i j}^{s d} Q_{i j}\right]+\left[\sum_{i j}\left(a_{i j}^{s d} R_{i}\right)+R_{d}\right]
$$

where $Q_{i j}$ is the transmission delay of the packets on lightpath $i j$, and $R_{i}$ is the processing delay at the electronic router of node $i$. In this paper, $Q_{i j}$ is determined by a $M / M / k_{i j}$ (where $k_{i j}$ shows the number of 
lightpaths between node pair $i j$ ) queuing system and $R_{i}$ by a $M / M / 1$ queueing system. Recall that we allow a multiple number of lightpaths between the node pair, and those lightpaths share same buffer (see Section 2). $Q_{i j}$ and $R_{i}$ are derived as follows.

$$
\begin{aligned}
Q_{i j} & =\frac{X_{l}}{l \cdot C-\lambda_{i j}}+\frac{1}{C} \\
R_{i} & =\frac{1}{\mu-\left(\lambda_{i j}+\delta_{i}\right)}
\end{aligned}
$$

where

$$
\begin{gathered}
X_{l}=\frac{p_{0}(l \rho)^{l}}{(1-\rho) l !} \\
\rho=\frac{\lambda_{i j}}{k_{i j} \cdot C} \\
p_{0}=\left\{\sum_{x=0}^{k_{i j}-1} \frac{\left(k_{i j} \rho\right)^{x}}{x !}+\frac{\left(k_{i j} \rho\right)^{k_{i j}}}{k_{i j} !\left(k_{i j}-\rho\right)}\right\}^{-1}
\end{gathered}
$$

Three kinds of packets arrive at the electronic router at node $i$; packets destined for node $i$, packets arriving at node $i$ from local access, and packets changing the lightpath at node $i$. Thus, $\delta_{i}$ is given by the following equation.

$$
\delta_{i}=\left[\sum_{j} \gamma_{j i}+\sum_{j} \gamma_{i j}+\sum_{s d, s \neq i, d \neq i} \sum_{j} a_{i j}^{s d} \gamma_{s d}\right]-\lambda_{i j}
$$

Note that $\lambda_{i j}$ is the flow rate of lightpath(s) between nodes $i$ and $j$. That is, we have

$$
\lambda_{i j}=\sum_{s d} a_{i j}^{s d} \gamma_{s d}
$$

Using equations (4) and (5), we finally obtain $l_{i j}$ as

$$
l_{i j}=\frac{\partial T}{\partial \lambda_{i j}}=\frac{1}{N(N-1)} \sum_{s=1}^{N} \sum_{d=1}^{N} a_{i j}^{s d} \alpha_{s d}
$$

where

$$
\alpha_{s d}=\frac{X_{k_{i j}}}{\left(l \cdot C-\lambda_{i j}\right)^{2}}+\frac{1}{\left(\mu-\left(\lambda_{i j}+\delta_{i}\right)\right)^{2}}
$$




\section{NUMERICAL EVALUATION AND DISCUSSIONS}

In this section, we evaluate our SHLDA by comparing with MLDA. In addition to MLDA, we also consider WLA (WDM Link Approach), where a WDM technology is only utilized for point-to-point link between adjacent IP routers.

\subsection{NETWORK MODEL}

As a network model, we consider 14-node NSFNET shown in figure 2. A traffic matrix given in [Ramaswami and Sivarajan, 1996] is used in numerical evaluation. Since the traffic matrix is given by a relative value, we introduce traffic scale $\alpha$, and actual traffic demands between nodes are given by a product of the traffic matrix and $\alpha$. We also assume the value of the given traffic matrix is represented in Gbps. We set the capacity of each wavelength to $10 \mathrm{Gbps}$. The packet processing capability of the electronic router, $\mu$, is represented in pps (packet per second) assuming that the mean packet size is 1,000 bits long.

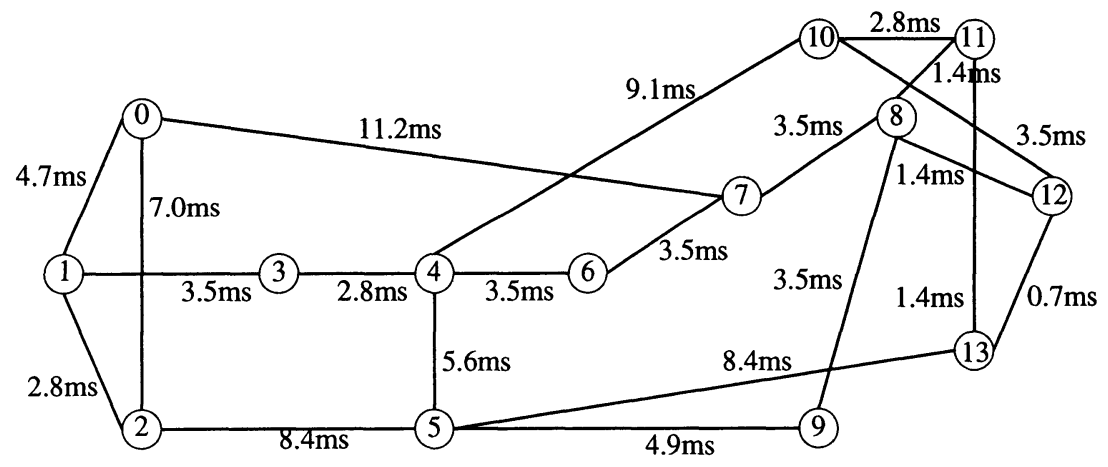

Figure 2 NSFNET

\subsection{NUMERICAL RESULTS AND DISCUSSIONS}

Figure 3 compares average delays obtained by three algorithms. The horizontal axis shows the traffic scale $\alpha$. In obtaining the figure, we set the number of wavelengths, $W$, to eight and the packet processing capacity of IP router, $\mu$, to $40 \mathrm{Mpps}$. In the figure, when the traffic scale $\alpha$ is small, we cannot observe significant differences among three algorithms, SHLDA, MLDA and WLA, and delays are suddenly increased as $\alpha$ becomes large in three algorithms. We also notice that our SHLDA is 


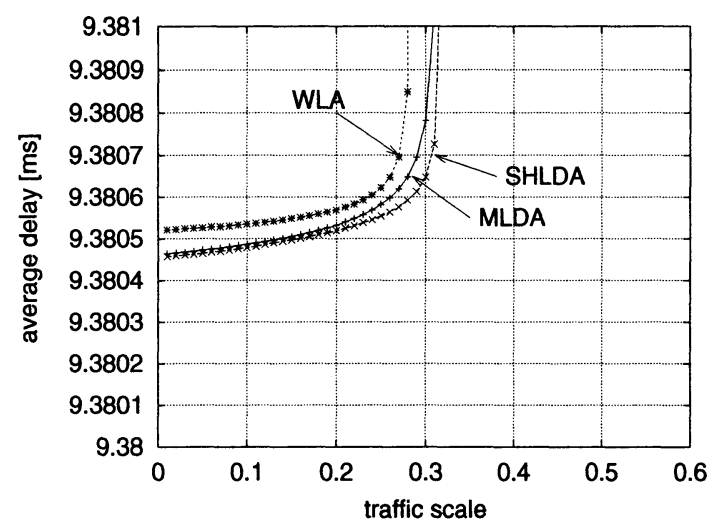

Figure 3 Average delay of each algorithm : $W=8, \mu=40 \mathrm{Mpps}$

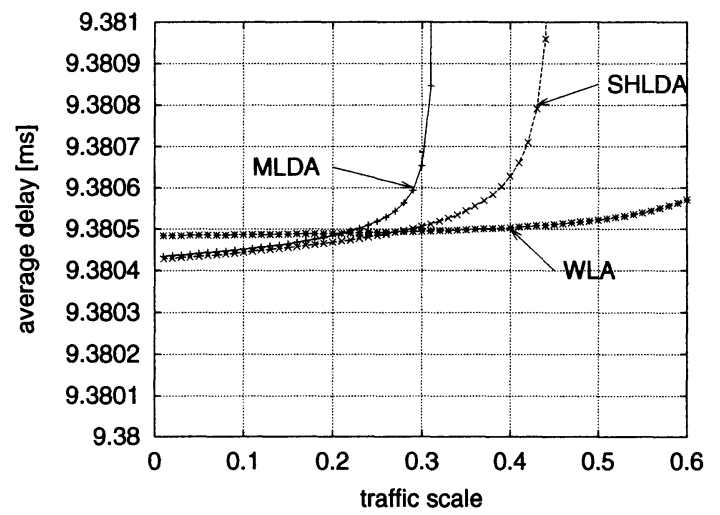

Figure 4 Average delay of each algorithm : $W=8, \mu=100 \mathrm{Mpps}$

slightly better than MLDA in terms of the maximum throughput which is a saturation point of the delays.

We next show the effect of increasing the packet forwarding capability of IP routers. Figure 4 shows the results by changing $\mu$ to $100 \mathrm{Mpps}$. For other parameters, same values in obtaining figure 3 are used. By comparing these two figures, we can observe that the maximum throughputs in SHLDA is increased as the IP router has enough capacity. However, improvement in the maximum throughput cannot be seen when we apply MLDA. To explain this, let us look at the nodal delays in more detail. Figures 5 and 6 show the processing delay and transmission delay dependent on $\alpha$. As expected, the effect of increasing the capability of the IP routers can be observed in these figures. As the processing delay is reduced with high capability of the IP router, the transmission delay beccomes the bottleneck of the network. Then, our SHLDA becomes superior to MLDA. 


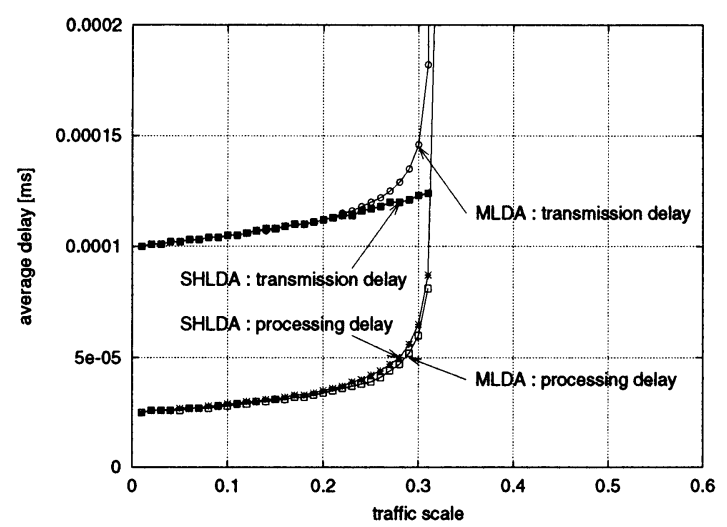

Figure 5 Average delay on node: $W=8, \mu=40 \mathrm{Mpps}$

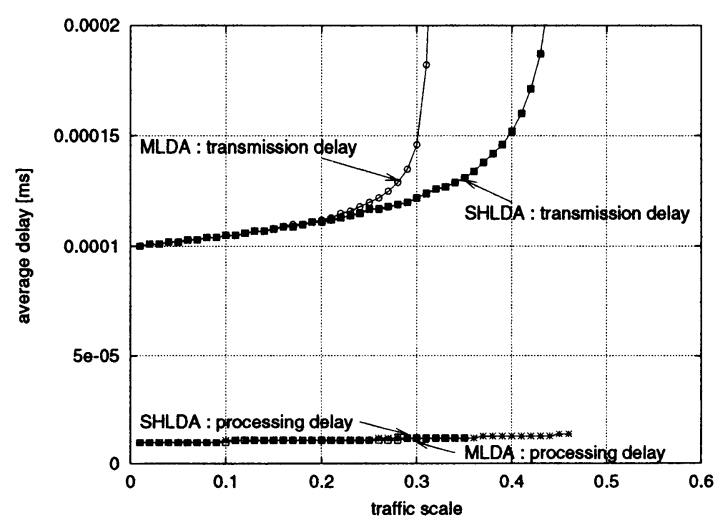

Figure 6 Average delay on node : $W=8, \mu=100 \mathrm{Mpps}$

We next set the number of wavelengths $W$ to twelve and $\mu$ to $40 \mathrm{Mpps}$. Results are shown in figure 7. By comparing figures 3 and 7, it is apparent that SHLDA exhibits the largest improvement on the maximum throughput. To see this more clearly, figure 8 presents components of delays in figure 7. By comparing figures 5 and 8 , we can see SHLDA can provide much improvements on the maximum throughput. In Figure 5 and 8 , we can see SHLDA does not show any difference in the transmission delays although the number of available wavelengths is increased from eight to twelve. On the other hand, the transmission delay by MLDA is decreased. Its reason can be explained as follows. SHLDA places lightpaths in a descending order of the product of the hop-count and traffic demand. As a result, a lightpath placed by SHLDA tends to utilize more links than the one by MLDA. Thus, MLDA can establish more lightpaths than SHLDA as the number of available wavelengths 


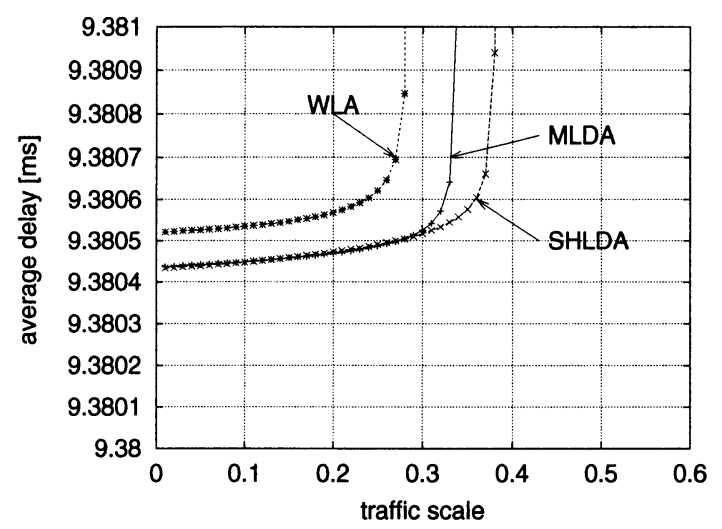

Figure 7 Average delay : $W=12, \mu=40 \mathrm{Mpps}$

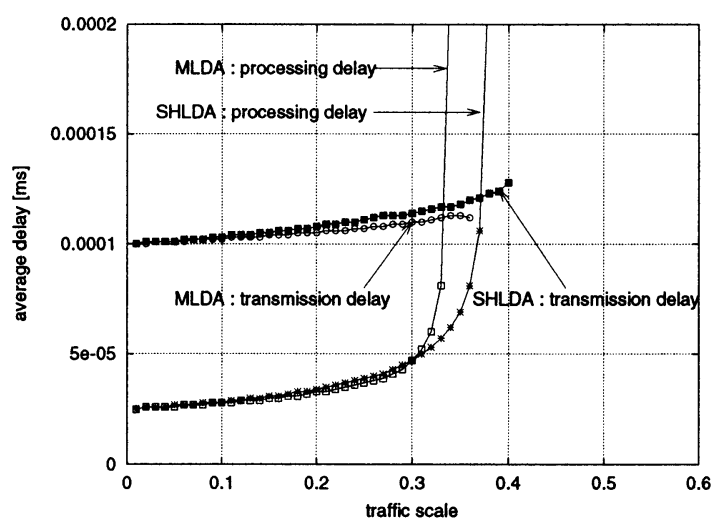

Figure 8 Delay on node : $W=12, \mu=40 \mathrm{Mpps}$

increases. It leads to decrease the transmission delay in MLDA as the number of wavelengths becomes large.

The processing delay at the IP router is reduced as the number of available wavelengths increases. Its effect is larger in SHLDA. As mentioned before, the lightpaths placed by SHLDA tend to utilize more physical links. It results in more reduction of electric processing in SHLDA than MLDA as the number of available wavelengths increases.

We last show the average delay obtained by our SHLDA by increasing the number of wavelengths. The results are plotted in figure 9 where we set $W=20$ and $\mu=40 \mathrm{Mpps}$. In this figure, SHLDA still shows higher throughput than MLDA, but the difference comparatively becomes smaller than the previous cases (figures 3 and 7). The reason is that by increasing the number of wavelengths, the logical topologies obtained by SHLDA or MLDA become close to a fully meshed network. Then, the advantage of SHLDA becomes smaller since SHLDA tries to 


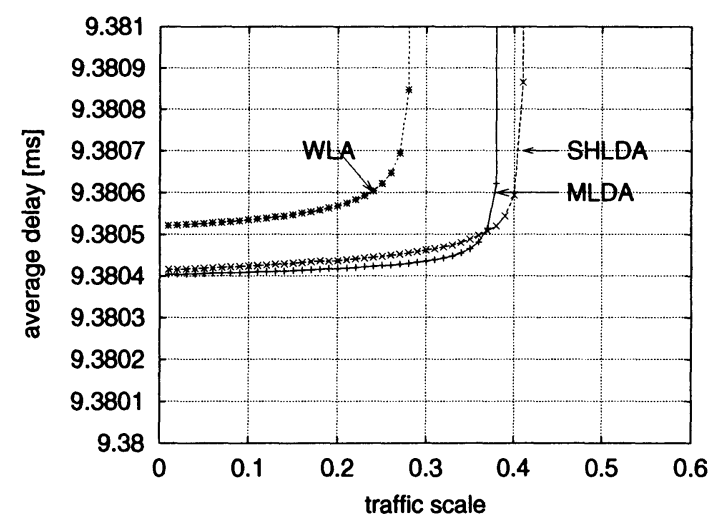

Figure 9 Average delay : $W=20, \mu=40 \mathrm{Mpps}$

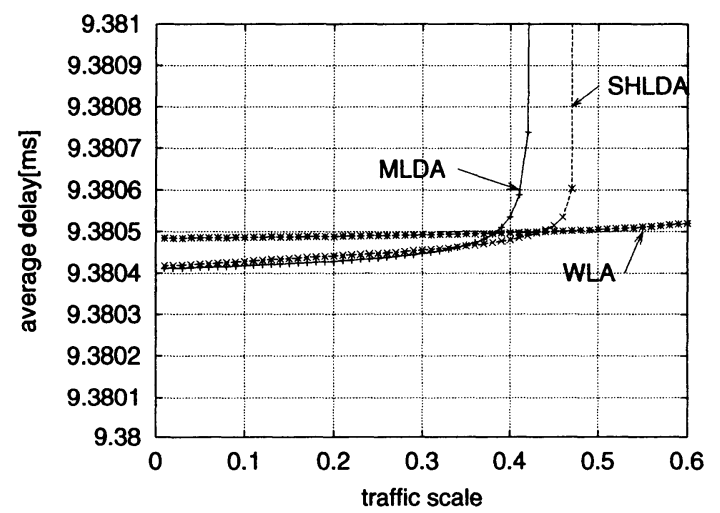

Figure 10 Average delay : $W=12, \mu=100 \mathrm{Mpps}$

reduce the traffic load on the IP router. We also show the case that $\mu$ is $100 \mathrm{Mpps}$. The result is plotted in figure 10 where we set $W=12$. By comparing figure 7 and 10, we can also observe the effectiveness of our SHLDA as well as in figure 3 and 4.

Lastly, we summarize the characteristics of WLA by observing figures 3, 4 and 7. By comparing figures 3 and 7, the improvement of the maximum throughput in WLA is very limited. This is because the processing delay at the electronic router is the primary bottleneck of the network, and henceforth, the effect of increasing the number of wavelengths cannot be seen. As one can easily imagine, the results of WLA is greatly improved as the capability of IP router becomes large (see figures 3,4 and 10). Only in that case, WLA is not a bad approach for IP over WDM networks. 


\subsection{INVESTIGATION ON ROUTING STABILITY}

We finally discuss our logical topology design algorithm from a viewpoint of the stability of IP routing. In IP networks, it is significant to avoid or at least to reduce unnecessary changing of the route, which is caused by dynamically changing traffic demand. To evaluate this, we examine the packet delays of first and second shortest end-to-end paths (lightpaths) prepared by our logical topology design algorithm. If those two values are close, the route of IP packets may frequently change against the traffic fluctuation.

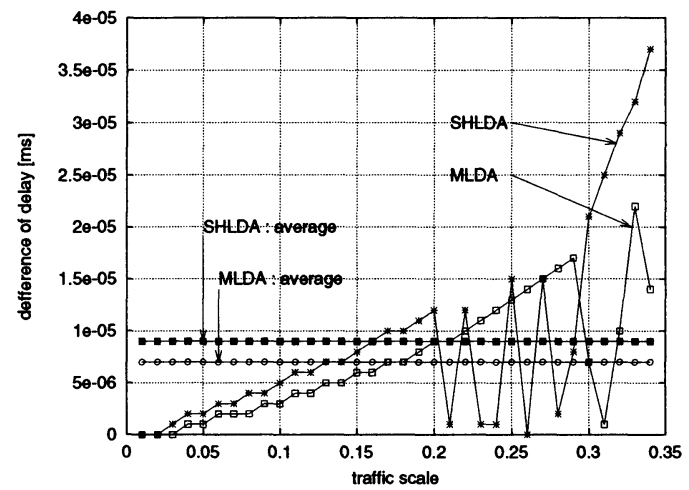

Figure 11 Route Stability : $W=8, \mu=40 \mathrm{Mpps}$

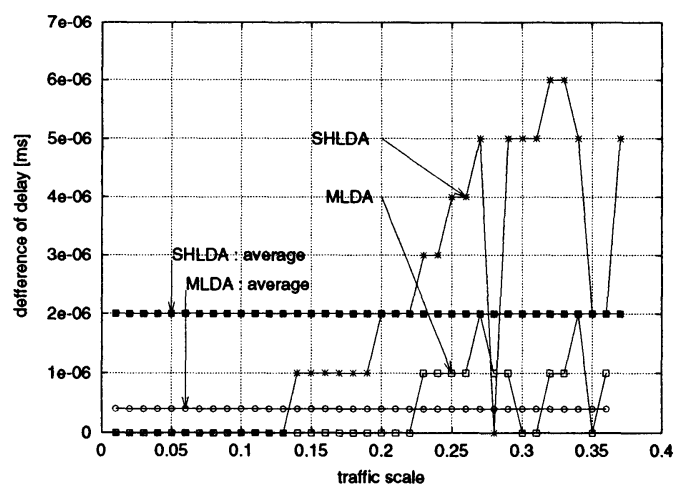

Figure 12 Route Stability : $W=12, \mu=40 \mathrm{Mpps}$

Let us introduce the metric $d_{s d}$ which defines the difference of delays of the first and second shortest routes between source node $s$ and destination node $d$. From all possible combinations of source and destination 
node pairs, we choose the smallest one as $d_{\min }$, i.e., $d_{\min }=\min _{s d}\left\{d_{s d}\right\}$. We consider that the design algorithm providing the larger $d_{\min }$ gives a higher routing stability. In figures 11 and 12 , we plot $d_{\min }$ obtained from SHLDA/MLDA as a function of $\alpha$, where the number of wavelengths $W$ is set to be eight and twelve, respectively. The processing capacity of the IP router, $\mu$, is identically set to be $40 \mathrm{Mpps}$ in both figures. The average of $d_{\min }$ is also shown in the figures. We can observe that when $W=8$ (figure 11), SHLDA is not very good especially when the traffic scale becomes large. However, it gives higher stability than MLDA when the number of wavelength to be twelve (figure 12) because of the design principle of our SHLDA.

The problem found in both of MLDA and SHLDA is that at several values of $\alpha, d_{\min }$ takes very small value. It is mainly because SHLDA as well as MLDA is a "one-way algorithm". That is, there are no step back operation in the algorithms; if the nodal delay is high, it tends to lead to the situation that the delay of the first shortest route becomes close to the delay of the second shortest one, since the nodal delay becomes domiant of the delay in such a region. We believe the situation can be avoided by reassembling the lightpaths to reduce the nodal delay, but it is one of our future research topics.

\section{CONCLUSION}

In this paper, we have proposed a new heuristic algorithm, SHLDA, to design a logical topology by considering the delay between nodes as an objective metric. Then, we have compared our proposed algorithm with conventional methods in terms of the average packet delay and throughput. The results have shown that SHLDA becomes effective when the number of wavelengths are small and the processing capacity of IP router is large. Furthermore, we have evaluated our proposed algorithm from a viewpoint of the routing stability. It is shown that SHLDA can obtain a stable network than MLDA.

However, in several values of the traffic scale, it has also been shown that SHLDA leads to the network having routes that can cause the routing instability than MLDA. To alleviate this problem, we need to reconfigure lightpaths in order to increase the routing stability. This is one of our future research topics. 


\section{References}

[D. O. Awduche and Coltun, 2000] D. O. Awduche, Y. Rekhter, J. D. and Coltun, R. (2000). Multi-protocol lambda switching: Combining MPLS traffic engineering control with optical crossconnects. draftawduche-mpls-te-optical-02.txt.

[Jamoussi, 2000] Jamoussi, B. (2000). Constraint-based LSP setup using LDP. draft-ietf-mpls-cr-ldp-04.txt.

[L.Fratta et al., 1973] L.Fratta, M.Gerla, and L.Kleinrock (1973). The flow deviation method: An approach to store-and-forward communication network design. Networks, 3:97-133.

[Mukherjee et al., 1996] Mukherjee, B., Banerjee, D., S.Ramamurthy, and Mukherjee, A. (1996). Some principles for designing a widearea WDM optical network. IEEE/ACM Transactions on Networking, 4(5):684-695.

[Ramaswami and Sivarajan, 1995] Ramaswami, R. and Sivarajan, K. N. (1995). Routing and wavelength assignment in all-optical networks. IEEE/ACM Transactions on Networking, 3:489-500.

[Ramaswami and Sivarajan, 1996] Ramaswami, R. and Sivarajan, K. N. (1996). Design of logical topologies for wavelength-routed optical networks. IEEE Journal on Selected Areas in Communications, 14:840851.

[R.Callon et al., 2000] R.Callon, A.Viswanathan, and E.Rosen (2000). Multiprotocol label switching architecture. draft-ieft-mpls-arch-07.txt.

[R.Callon et al., 1999] R.Callon,

G.Swallow,

N.Feldman, A.Viswanathan, P.Doolan, and A.Fredette (1999). A framework for multiprotocol label switching. draft-ietf-mpls-framework-05.txt.

[R.Dutta and G.N.Rouskas, 2000] R.Dutta and G.N.Rouskas (2000). A survey of virtual topology design algorithms for wavelength routed optical networks. Optical Network Magazine, 1(1):73-89. 
[R.Ramaswami and Sivarajan, 1995] R.Ramaswami and Sivarajan, K. N. (1995). Design of logical topologies for wavelength-routed all-optical networks. In IEEE Infocom, pages 1316-1325. 